\title{
Composición, estacionalidad y hábitat de los peces de la quebrada Cristales, afluente del Río la Vieja, Alto Cauca, Colombia.
}

\author{
Composition, periodism and habitat of fishes in the cristales creek, Vieja River \\ drainage, Upper Cauca, Colombia
}

\author{
Roberto J. García Alzate*, Carlos A. García-Alzate** y Álvaro Botero-Botero****
}

Recibido: Octubre 8de 2008

Aceptado: Enero 29 de 2009

Correspondencia: Laboratorio de Ictiologia, Universidad del Quindio. Avenida Bolivar calle 12 norte Armenia Quindio. Email: cagarcia@uniquindio.edu.co

\section{RESUMEN}

Se estudió la composición, estacionalidad y hábitat de los peces de la Quebrada Cristales, afluente terciario del río La Vieja en el Alto Cauca, Colombia, desde junio 2004 hasta enero, 2005. Se capturaron 16 especies de cuatro órdenes y cinco familias; las capturas se efectuaron con métodos estándares en 14 estaciones de muestreo a lo largo de la quebrada. Brycon henni fue constante en la quebrada para ambos periodos climáticos y en las zona baja de la quebrada. Poecilia caucana es la especie con más alto porcentaje de ocurrencia para ambos periodos y la única especie constante para la zona alta. Apteronotus sp., Argopleura magdalenensis, Bryconamericus caucanus, Chaetostoma fischeri, Characidium caucanum, Imparfinis nemacheir, Lasiancistrus caucanus, Rhamdia quelen, y Roeboides dayi fueron especies accidentales y solo se capturaron a medida en cercanías a la desembocadura, observándose únicamente para la zona media y baja de la quebrada. Se evaluaron características fisicoquímicas y del hábitat. Muchas especies se encontraron asociadas a microhabitats con abundante vegetación ribereña y macrófitas acuáticas.

Palabras claves: Alto río Cauca, Colombia, Peces, Quebrada Cristales, comunidades.

\begin{abstract}
The composition, seasonality and habitat of the fish from the Cristales creek, tertiary tributary of the La Vieja river, upper Cauca, Colombia, was studied from June 2004 to January 2005. Sixteen species of four orders and five families were captured; the captures were made with standard methods through 14 stations distributed in the creek. Brycon henni species were constant to both climatic periods and in the low area of the creek. Poecilia caucana were the species with higher occurrence percentage for both periods and the only constant species for the high area. Apteronotus sp., Argopleura magdalenensis, Bryconamericus caucanus, Chaetostoma fischeri, Characidium caucanum, Imparfinis nemacheir, Lasiancistrus caucanus, Rhamdia quelen, and Roeboides dayi were accidental and they were captured when we were very close to the outlet creek, and these species were observed in the half and low parts of the creek. Physicochemical and habitat characteristics were evaluated. Many species were associated to microhabitats with abundant riverside vegetation and aquatic macrophyties.
\end{abstract}

Key Words: Upper river Cauca, Colombia, fish, Cristales creek, communities.

\section{INTRODUCCIÓN}

$\mathrm{E}$ l estudio de la estructura de las comunidades de peces tiene dos enfoques principales: el primero es el análisis de los factores que determinan el número de las diferentes clases de organismos que se encuentran juntos en un hábitat y su abundancia relativa, el segundo es el análisis de los porcentajes de la energía y nutrientes que son utilizados dentro del hábitat, tamaño de la biomasa y la estructura de los organismos allí presentes. Los dos enfoques están muy relacionados, pero las interacciones en la naturaleza son poco conocidas (Wootton, 1992), más aun en

\footnotetext{
* Laboratorio de Física, Instituto de Biología Experimental “IBE”, Universidad Central de Venezuela. Caracas Venezuela.

** Laboratorio de Ictiología. Programa de Biología. Universidad del Quindío

*** Fundación Neotrópica-Colombia, Armenia, Quindío, Colombia.
} 
el neotropico donde las comunidades de peces de agua dulce son característicamente ricas en especies y presentan complejas inter-relaciones entre sus componentes (LoweMcConnell, 1987), además con pocos estudios.

Para el propósito de este trabajo se definen las comunidades de peces como el conjunto de especies en cada uno de los ecosistemas muestreados. Son entonces unidades de carácter practico, que representan un segmento del ecosistema relativamente uniforme y que son sencillamente el resultado de la técnica de muestreo (Margalef, 1982). En este estudio se consideran como componentes primarios de los ecosistemas, la composición entendida como la identidad y variedad de elementos (Noss, 1990).

Para el Alto Cauca es poca la información que se tiene sobre la composición y estacionalidad de la comunidad de peces, salvo pocas excepciones como los trabajos realizados en comunidades por Cardona et al. (1998), Jiménez et al. (1998), Lehmann (1999), Román-Valencia (1993) y RománValencia et al. (1999).

El objetivo fue determinar la composición y estacionalidad de la comunidad de peces de la quebrada Cristales, afluente del río La Vieja, Alto Cauca, Colombia.

\section{MATERIALES Y MÉTODOS}

\section{Área de estudio.}

La quebrada Cristales es un afluente de tipo terciario del río La Vieja, se encuentra ubicada en la zona Centro Occidental del departamento del Quindío, involucrando en su recorrido a los municipios de Armenia y La Tebaida. Nace en el sector del sur de Armenia (4 $30^{\prime} 41^{\prime \prime} \mathrm{N}$ y $75^{\circ} 42^{\prime} 32^{\prime \prime} \mathrm{O}$ a 1.475 $\mathrm{msnm})$, en una zona caracterizada por una topografía ondulada, el área total de la microcuenca es de $91.43 \mathrm{~km} .2$, con una pendiente promedio de $1.25 \%$, tiene una longitud de $33.66 \mathrm{~km}$. hasta su desembocadura en el Río La Vieja (Vargas, 1997) en el sector de Valle de Pisamal ( 4 24' 53" N y 75은 15 " O a $1.047 \mathrm{msnm}$ ) (figura 1).

La quebrada Cristales fue dividida en tres zonas (alta, media y baja), de acuerdo con las características geomorfológicas, físicas, químicas; descritas por el Grupo de investigaciones de ingeniería civil de la Universidad del Quindío (CEIFI, 2003). Cada zona se dividió a su vez en tres puntos equidistantes, sumando nueve estaciones de muestreo. Además se incluyeron cinco estaciones anteriormente trabajadas por el CEIFI (2003), donde dos pertenecen a la zona alta, una a la parte media y dos a la zona baja, sumando en total 14 estaciones de muestreo.

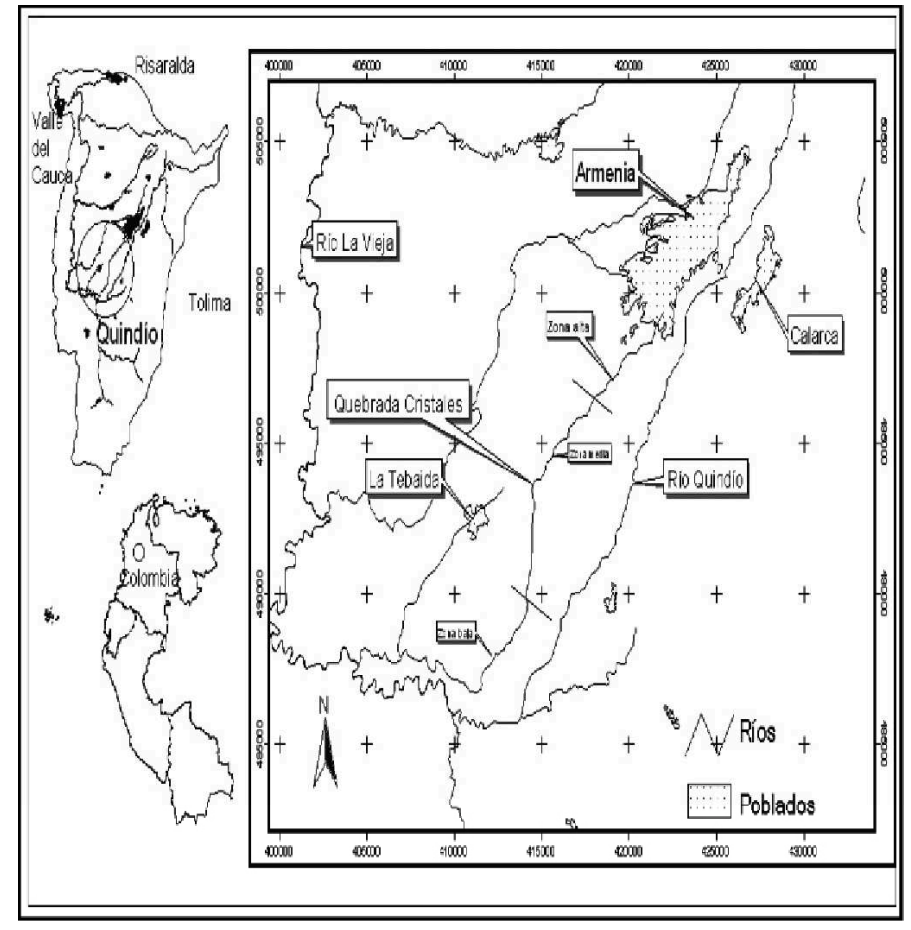

Figura 1. Área de estudio, se muestra la Parte Alta, Baja y Media de la quebrada Cristales.

Se caracterizó el área de estudio por estación teniendo en cuenta la vegetación ribereña dominante, sustrato de la quebrada, afluentes aledaños, altitud, velocidad de la corriente. Se midieron In situ temperatura del agua y temperatura ambiente, oxigeno disuelto y porcentaje de saturación del agua $(\mathrm{mg} / \mathrm{l})$, con oxímetro digital, $\mathrm{pH}$ con potenciómetro digital, y humedad relativa con termohigrómetro. Además, para los periodos de sequía (agosto) y lluvia (noviembre), se tomaron muestras de agua de la estación baja, para determinar otras variables en el laboratorio de aguas de la universidad del Quindío como: demanda bioquímica de oxigeno (DBO5), demanda química de oxigeno (DQO), alcalinidad, sólidos suspendidos y sólidos disueltos, se determinaron de acuerdo con la metodología recomendada por la APHA (1992) y Wetzel \& Likens (2000).

Se realizaron colectas mensuales desde junio 2004 hasta enero 2005, abarcando los periodos de lluvias bajas (diciembre-enero y julio-agosto) y lluvias altas (septiembrenoviembre). Se ejecutaron en las horas del día, y para tener en cuenta las especies de hábitos nocturnos, se realizaron dos salidas nocturnas en cada periodo.

Para captura de los individuos fueron usadas redes de arrastre de 3,6 $\mathrm{m}$ de ancho por 1,6 $\mathrm{m}$ de alto, (con un tamaño de malla $0,5 \mathrm{~cm}$ de diámetro), nasa de $80 \mathrm{~cm}$ de diámetro por $60 \mathrm{~cm}$ de profundo (con un tamaño de malla de $0.5 \mathrm{~cm}$ entre nudos) y atarraya de $3,40 \mathrm{~m}$ de diámetro por $3.10 \mathrm{~m}$ de alto 
(con un ojo de malla de $1,5 \mathrm{~cm}$ ), los muestreos abarcaron los diferentes microhábitas que se presentaran en la estación: remanso, corriente, o ambos, vegetación ribereña. La frecuencia fue de tres arrastres por sitio (uno con la corriente, otro en contra y uno de orilla a orilla), para la captura de los individuos de mayor tamaño se utilizo la atarraya, con frecuencia de un lance por sitio; lo anterior para evitar el fuerte disturbio que genera el lance de la atarraya en la captura de peces en el cuerpo de agua; esta estandarización en los métodos de muestreo permitió que las capturas y estaciones no fueran causa de variación.

Durante las faenas de pesca los peces capturados fueron depositados en un recipiente con agua para evitar recapturas y culminada la faena se identificaron In situ hasta nivel taxonómico mas bajo posible y posteriormente fueron liberados. Los ejemplares que no se lograron identificar, se les registró la coloración en vivo y conservaron en formaldehido al $10 \%$ y se trasladaron al laboratorio de biología de la Universidad del Quindío para su posterior identificacion con base en claves, descripciones y registros (Román-Valencia 1995, 2003; Maldonado-Ocampo et al., 2005; Ruiz-C y Román-Valencia 2006 a, b), y la colaboración del museo de Biología de la Universidad Central de Venezuela (MBUCV). Las muestras colectadas fueron depositadas en el museo de Biología de la Universidad Central de Venezuela (MBUCV), y en el Laboratorio de Ictiología de la Universidad del Quindío (IUQ). Para cada estación muestreada se midió, la constancia de ocurrencia de las especies, Uieda (1984): $\mathrm{C}=\left(\mathrm{Pi}^{\prime} \mathrm{P}^{\prime}\right) \times 100$, donde: $\mathrm{C}$ es la constancia de ocurrencia, Pi número de individuos de la especie i y $\mathrm{P}^{\prime}$ total de individuos capturadas en la estación $\mathrm{j}$. Los valores de $\mathrm{C}$ permiten clasificar las especie como: constante (C: mayor de 50 ), accesoria (C: entre 25 y 50 ), accidental (C: menor de 25).

\section{RESULTADOS}

\section{Características del Hábitat.}

En la quebrada se encuentran tres zonas claramente diferenciadas: la zona alta con una vegetación ribereña conformada por bosques de Guadua angustifolia y algunos remanentes boscosos. El sustrato conformado principalmente por arena, lodo, materia orgánica en descomposición y cantos rodados, el oxigeno disuelto fue bajo $(3,2 \mathrm{mg} / \mathrm{l})$, la temperatura promedio del agua $20^{\circ} \mathrm{C}$, el pH promedio 4,8 (tabla 1), en esta zona la quebrada recibe la descargas de aguas servidas de los barrios y fincas aledañas lo que la convierte en una zona altamente eutroficada. La zona media de la quebrada presentó las siguientes características: vegetación ribereña conformada por bosques deG. angustifolia y bosques en sucesión secundaria en la gran mayoría de la ribera, sustrato conformado principalmente por arena, cantos rodados y materia orgánica de origen vegetal en descomposición, el oxigeno disuelto fue alto $(7,5$ $\mathrm{mg} / \mathrm{l})$, la temperatura promedio del agua de $21,5^{\circ} \mathrm{C}$, el pH 6,4 (tabla 1), en este sector el cauce presenta dos biotopos claramente definidos: los remansos que se caracterizan por presentar forma de pozo con velocidades bajas de corriente $y$ deposición de lodos y arenas, y los rabiones los cuales son zonas de corrientes rápidas y bajas profundidades caracterizadas principalmente por sustratos rocosos. En la zona baja la quebrada entra en el valle de Maravelez y Pisamal, en donde presenta áreas de inundación o pequeñas madreviejas, el espejo laminar recibe totalmente la entrada de luz, se encuentra asociación de macrófitas acuáticas como mataandrea Hedychium coronarium (Zingibereraceae) y Elodea sp. que brinda disponibilidad de microhábitat a la fauna acuática, su sustrato es inestable y conformado principalmente por sedimentos finos de arena, y fango, presentó un oxigeno disuelto bajo $(5,5 \mathrm{mg} / \mathrm{l})$, la temperatura promedio del agua $24,5^{\circ} \mathrm{C}$, el pH promedio 7,3 (tabla 1), las aguas en esta zona fueron predominantemente turbias.

Tabla 1. Valores promedio de las variables físicas y químicas en las tres zonas de la quebrada Cristales, Alto Cauca, Colombia.

\begin{tabular}{|c|c|c|c|}
\hline \multirow[b]{2}{*}{ Variables } & \multicolumn{3}{|c|}{ Zona de la Quebrada } \\
\hline & Parte alta & arte me & arte baja \\
\hline Temperatura del agua $\left({ }^{\circ} \mathrm{C}\right)$ & 20 & 21.5 & 24.5 \\
\hline Oxigeno disuelto $\left(\mathrm{mg} / \mathrm{LO}_{2}\right)$ & 3.2 & 7.5 & 5.5 \\
\hline Potencial de hidrogeniones(PH) & 4.8 & 6.4 & 7.3 \\
\hline $\begin{array}{c}\text { Demanda Bioquímica de } \\
\text { Oxigeno( }\left(\mathrm{mg}^{\prime} / \mathrm{LO}_{2}\right)\end{array}$ & 48.5 & 7.5 & 8.5 \\
\hline $\begin{array}{c}\text { Demanda Química de } \\
\text { Oxigeno }\left(\mathrm{mg}^{\prime} / \mathrm{LO}_{2}\right)\end{array}$ & 70 & 19 & 26 \\
\hline Sólidos Totales (mg/L) & 230 & 133 & 167 \\
\hline Sólidos Disueltos (mg/L) & 213 & 85 & 136 \\
\hline Sólidos Suspendidos (mg/L) & 17 & 48 & 31 \\
\hline
\end{tabular}

La temperatura del agua, porcentaje de saturación del oxigeno, oxigeno disuelto, $\mathrm{pH}$, humedad relativa, velocidad de la corriente, ancho, profundidad, sólidos totales, suspendidos y disueltos Aumentaron en invierno (tabla 2), por lo que pueden estar influenciados por procesos de lixiviación y escorrentía que sufre la cuenca con las lluvias, iguales resultados fueron encontrados por Botero y RománValencia (2005) para la quebrada Tinajas, García-Alzate \& Román-Valencia (2008) para la quebrada el Indio y GarcíaAlzate et al., (2007) para las quebradas Doña Juana y Villa Paola.

\section{Comunidad de peces.}

En total se hallaron 16 especies que representan el $23,8 \%$ de las especies nativas registradas hasta el momento para el alto 
Cauca (Ortega-Lara et al., 2006), agrupadas en cuatro órdenes, cinco familias y 16 especies (tabla 3), de las cuales Characidae con el $43,7 \%$ del total de especies para la quebrada. La especie Brycon henni fue la más dominante con un $59,63 \%$, seguida de Poecilia caucana con una dominancia del $23,40 \%$.

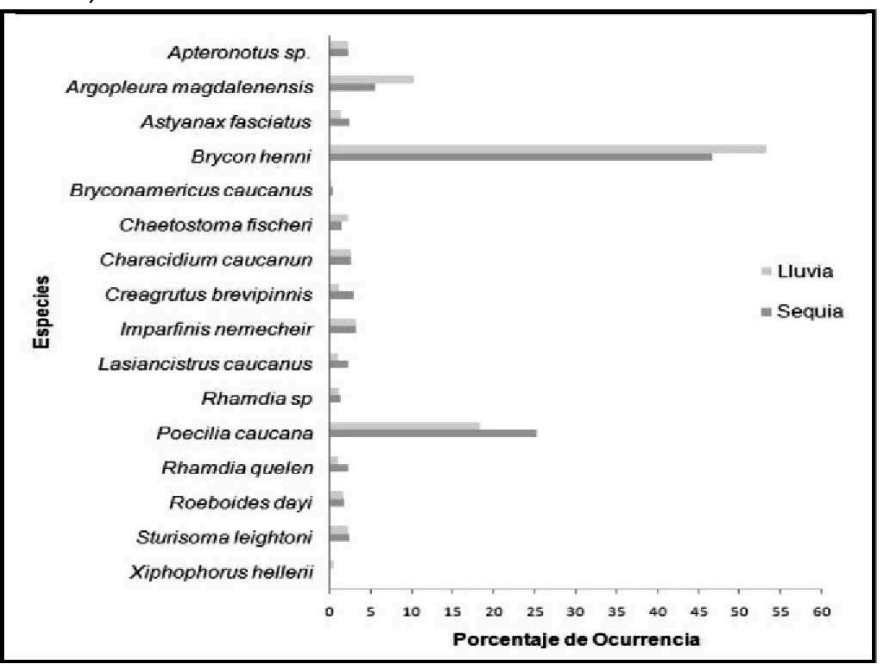

Figura 2. Constancia de ocurrencia de las especies colectadas en la quebrada Cristales, periodos sequía y lluvia.

De las 16 especies colectadas solo Brycon henni fue constante en la quebrada para ambos períodos climáticos, con un aumento leve en la temporada de lluvias (figura 2), y se colecto en la zona baja de la quebrada, mientras que el resto de especies fueron accidentales para los periodos de lluvia y sequía, siendo de este grupo Poecilia caucana la especie con más alto porcentaje de ocurrencia en ambos periodos (figura 2 ), a su vez $P$. caucana es la única especie constante en la zona alta con una tendencia a la agregación (figura 3).

Tabla 2. Variables físicas y químicas de dos periodos climáticos, en la quebrada Cristales, Alto Cauca, Colombia (año 2004).

\begin{tabular}{|l|c|c|}
\hline \multirow{2}{*}{\multicolumn{1}{c|}{ Variables }} & \multicolumn{2}{|c|}{ Periodo } \\
\cline { 2 - 3 } & Sequía (Prome dio) & Lluvia (Promedio) \\
\hline Temperatura del agua $\left({ }^{\circ} \mathrm{C}\right)$ & 20 & 22,6 \\
Temperatura delambiente $\left({ }^{\circ} \mathrm{C}\right)$ & 17,2 & 23,6 \\
Porcentaje de saturación $(\%)$ & 91 & 97 \\
Oxigeno disuelto (ml/l) & $5,4(2,2-8,3)$ & $7,3(2,5-8,5)$ \\
pH & 6,36 & 7,7 \\
Humedad relativa $(\%)$ & 92 & 94 \\
Sustrato & Fango y detritos & Arena y detritos \\
Color & Oscuro & Marrón \\
Velocidad de la corriente (m/s) & 0,25 & 0,84 \\
Ancho (m) & 2,95 & 4,06 \\
Profundidad (m) & 0,6 & 1,2 \\
D.B.O (mg/l) & 14 & 9 \\
D.Q.O (mg/l) & 30 & 23 \\
Sólidos totales (mg/l) & 70 & 99 \\
Sólidos suspendidos $(\mathrm{mg} / \mathrm{l})$ & 3 & 5 \\
Sólidos disueltos (mg/l) & 69 & 96 \\
\hline
\end{tabular}

Argopleura magdalenensis aumentó su constancia de ocurrencia para periodo de lluvias, mientras que desciende en periodo de sequía, Roeboides dayi sólo se colectó en la zona baja de la quebrada como una especie accidental (Figura. 3), y presentando un incremento en el periodo de Iluvia.

Además, Apteronotus sp., Imparfinis nemacheir, Rhamdia quelen, Rhamdia sp., Chaetostoma fischeri y Lasiancistrus caucanus fueron especies accidentales y se capturaron a medida que se baja en el cuerpo de agua, viéndose representadas en mayor constancia en la parte baja, asociados a microhábitas con abundante vegetación ribereña como pastos para ganadería (Poacea), $H$. coronarium, y Elodea sp. y sustratos conformados por arena, piedra y lodos, Lasiancistru caucanus fue capturado predominantemente en la sitios con alta presencia de $H$. coronarium, ubicándose de bajo de las hojas que están sumergidas, Apteronotus sp., Imparfinis nemacheir, fueron asociados principalmente a pasto para la ganaderia, mientras que Bryconamericus caucanus se colectó únicamente en la parte baja (figura 3).

Tabla 3. Lista de las especies de la quebrada cristales, Alto Cauca, Colombia.

\begin{tabular}{|c|c|}
\hline Especies & Nombre común \\
\hline \multicolumn{2}{|l|}{ Clase: Osteichthyes } \\
\hline \multicolumn{2}{|l|}{ Orden: Characiformes } \\
\hline \multicolumn{2}{|l|}{ Familia:Characidae } \\
\hline Argopleura magdalenensis & Sardima \\
\hline Astyanax fasciatus & Sardina colirroja \\
\hline Bryconamericus caucamus & Sardima \\
\hline Brycon henni & Sardima \\
\hline Creagrutus brevipinnis & Cabeza de candado \\
\hline Characidium caucamum & Sardima \\
\hline Roeboides dayi & Juan viejo-Jorobado \\
\hline \multicolumn{2}{|l|}{ Orden: Gymnotiformes } \\
\hline \multicolumn{2}{|l|}{ Familia:Apteronotidae } \\
\hline Apteronotus sp. & Boca de perra \\
\hline \multicolumn{2}{|l|}{ Orden: Siluriformes } \\
\hline \multicolumn{2}{|l|}{ Familia: Heptapteridae } \\
\hline Rhamdia sp & Barbudo \\
\hline Imparfinis nemacheir & Barbudo \\
\hline Rhamdia quelen & Bardudo \\
\hline \multicolumn{2}{|l|}{ Familia: Loricariidae } \\
\hline Chaetostoma fischeri & Corroncho \\
\hline Lasiancistrus caucamus & Corroncho \\
\hline Sturisoma leightoni & Corroncho \\
\hline \multicolumn{2}{|l|}{ Orden: Cyprinodontiformes } \\
\hline \multicolumn{2}{|l|}{ Familia:Poeciliidae } \\
\hline Poecilia caucana & Guppy \\
\hline Xiphophorus helleri & Espada (Introducido) \\
\hline
\end{tabular}




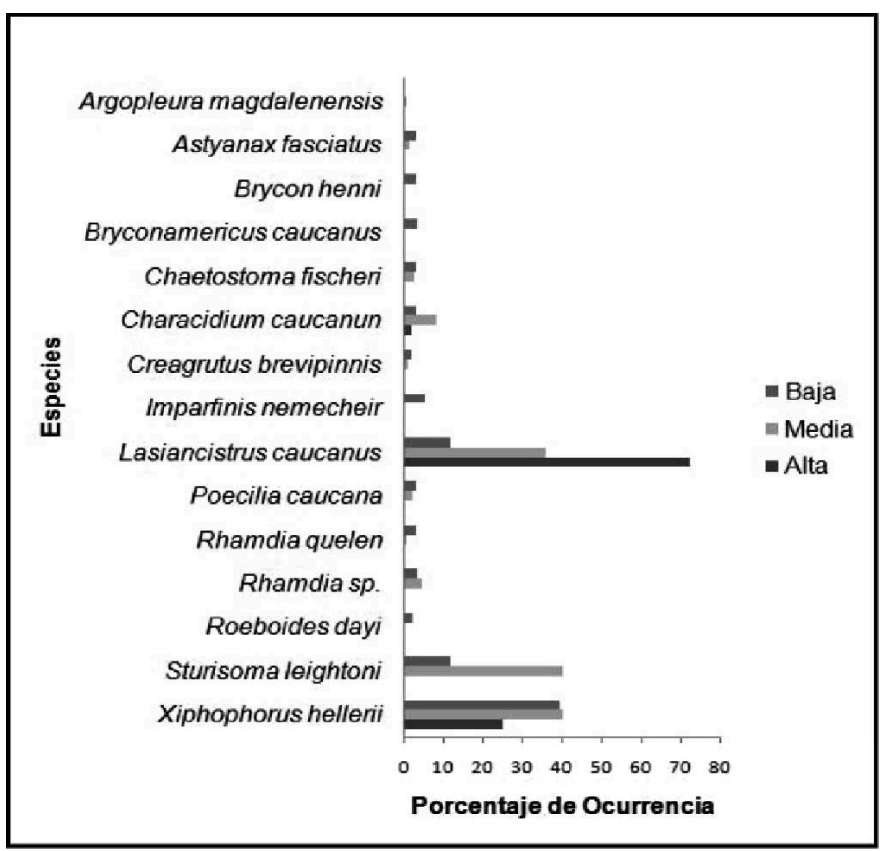

Figura 3. Constancia de ocurrencia de las especies, en la quebrada Cristales Alto Cauca Colombia.

\section{DISCUSIÓN}

Los resultados encontrados en el presente trabajo con relación a la composición de la comunidad de peces, muestran en general los patrones registrados para la íctiofauna Neotropical en cuanto a que es mucho más rica en número de especies pertenecientes a los ordenes Characidae y Siluriformes, conformando la mayoría de las especies en una comunidad (Cala, 1990; Lowe-McConnell, 1987), además resultados similares fueron reportados para otras comunidades del alto Cauca por (Cardona et al., 1998; Jiménez et al., 1998; Lehmann, 1999; Román-Valencia, 1993).

Brycon henni presentó una amplia distribución en la cuenca del río La Vieja, pudiéndose capturar en la mayorías de ríos y quebradas de la cuenca (Román-Valencia, 1993), la especie esta presente en zonas con concentraciones altas de oxigeno (7-10 mg./l), prefiriendo aguas claras, ubicándose en el día en las zonas finales de los charcos para la captura de alimentos (Builes y Uran, 1974), esto puede explicar que la especie solo sea constante en la parte baja donde se presentan los mayores valores de oxigeno (tabla 3 ), y el cauce presenta una mayor heterogeneidad espacial. Para la quebrada fueron capturados individuos tanto adultos con longitud estándar mayor de $16 \mathrm{~cm}$ (Builes y Uran, 1974) como juveniles durante todo el año. La no captura de individuos adultos de $B$. henni reportada por Jiménez et al., (1998), para la quebrada San Pablo, Alto Cauca, podría estar influenciados mas por el método de captura y no por migraciones reproductivas que ellos reportan.
Poecilia caucana es la única especie constante en la zona alta, pues en esta zona proliferan aguas lentas y sustrato arenoso y fangoso y niveles bajos de oxigeno y agua altamente contaminadas (Nagles y Vargas 2003), iguales resultados fueron reportados para esta especie por Jiménez et al. (1998) dado que la especie prefiere este tipo de aguas y sustratos que favorecen sus supervivencia.

La tendencia a la agregación de individuos de esta especie también ha sido reportada para especies del mismo genero, como Poecilia gillii y a su vez esta especie presenta una dieta basada en fango y algunas algas filamentosas y diatomeas (Bussing, 1993) al igual que $P$. caucana que para la zona de la cuenca del río La Vieja presenta una dieta predominantemente alguívora (microalgas) (Cuartas, 2005), dado lo anterior $P$. caucana es una especie euritópicas, con una plasticidad ecológica que le facilitan explotar y tolerar los tensores ambientales de la parte alta, posiblemente se esté alimentando de las algas, fango y insectos del orden Chironomidae que prolifera en esas áreas (Nagles y Vargas, 2003). Además otra adaptación característica de los poecilidios y de $P$. caucana es ser una especie vivípara y de reproducirse durante todo el año (Cuartas, 2005), que le confiere la característica no exponer ovocitos a la degradación generada por los tensores ambientales y mantener los niveles poblacionales siempre altos durante todo el año.

El aumento de la constancia de ocurrencia en el periodo de lluvias de $A$. magdalenensis es producto de migraciones de tipo reproductivo que realiza la especie desde el río La Vieja a pequeños afluentes, pues los ejemplares sexualmente maduros de esta especie al inicio de las lluvias se desplazan a las corrientes secundarias de la cuenca, las cuales les proporcionan las condiciones adecuadas para lograr el éxito reproductivo y evitar la competencia interespecífica por el espacio (Román-Valencia y Perdomo, 2004).

Roeboides dayi solo se presentó en la parte baja como una especie accidental, pues la especie en el alto Cauca alcanza solo los 1100 m.s.n.m (Jiménez et al., 1998; Román-Valencia et al., 1999, 2003) y habita en ríos y quebradas de tipo secundario, donde prolifera un gradiente de charcos $y$ rápidos y un sustrato de piedras, arena, y material vegetal en descomposición, y riberas con vegetación asociada (RománValencia et al., 2003). El aumento de la constancia en la época de lluvias puede ser influenciado por aumento en el numero de individuos producto del desove en verano, pues la especie presenta un desove en invierno y otro en verano (RománValencia et al., 2003) y no a productos de migraciones reproductivas, pues la especie no presenta este tipo de migraciones (Román-Valencia et al., 2003). 
La captura de L. caucanus en estaciones con alta presencia de $H$. coronarium, y ubicados bajo las hojas sumergidas esta dado porque en estos microhábitas se asocian algas epifititas que son uno de los principales ítem alimenticios de $L$. caucanus (Zamudio, 2004). Apteronotus. sp., I. nemacheir, $R$. quelen y fueron asociados principalmente a pasto Kikuyo (Poacea), encontrando allí disponibilidad de refugios y alimento. Pues las partes sumergidas de las macrófitas acuáticas forman un hábitat complejo representando un importante refugio para los peces, especialmente contra depredadores (Sánchez-Botero et al., 2003). La presencia de estos grupos de Siluriformes también puede estar relacionada por los hábitos nocturnos y la tendencia del grupo a explorar ambientes de fondo (Lowe-McConnell, 1987).

Por otra parte, el aumento gradual de la constancia de ocurrencia para las zonas (alta, media y baja), se encuentra determinada a medida que disminuye la altitud, cumpliéndose esto también para los trabajos realizados por
Jiménez et al., (1998) y Román-Valencia et al., (1999). Y aumenta la complejidad el sistema acuático a medida que la quebrada cobra tamaño y entidad, brindando así mayor disponibilidad de hábitats, microhábitat y refugios pues en la zona baja posee abundante vegetación asociada y pequeñas zonas de inundación, además de presentar una dinámica trófica propia por su condición de riachuelo. Por otra parte, la zona baja de la quebrada, presentó mejores condiciones fisicoquímicas, que facilitan el desarrollo de la ictiofauna.

Nebiolo (1987) y Román-Valencia (1998), plantearon que el aumento del número de especies a medida que disminuye la altura, refleja el incremento del porcentaje en la constancia de ocurrencia en el tramo medio y bajo de una cuenca, debido al grado de complejidad del ambiente, a la formación y a la disponibilidad de nutrientes, esto fue corroborado para la Quebrada Cristales, además a medida que la quebrada desciende los índices de calidad de agua mejoran (Nagles y Vargas, 2003) lo que favorece las comunidades de peces.

\section{AGRADECIMIENTOS}

Los autores agradecen a Jorge Ivan Sanchez (Brasil), Otto Castillo (Venezuela), Donald Taphorn (Venezuela) por la lectura y sugerencias del manuscrito. Al museo de biología de la Universidad Central de Venezuela (MBUCV), y al Laboratorio de Ictiología de La Universidad del Quindío (IUQ) por la colaboración en la determinación de los peces.

Carlos García T, Jairo Torres, y Johanny Aguillon por la colaboración en el trabajo de campo. Al laboratorio de Biología de la Universidad del Quindío por el préstamo de materiales y equipos.

\section{BIBLIOGRAFÍA}

APHA, AWWA, WPCF. 1992. Métodos normalizados para el análisis de aguas potables y residuales. Ed. Díaz de Santos,S.A. Barcelona (España). p.385.

Builes J. y Uran A. 1974. Estudio del cíclo sexual de la Sabaleta Brycon henni. Su comportamiento y fecundación artificial. Revista Actualidades Biologicas. 3:2-12.

Bussing A. 1993. Fish communities and environmental characteristics of a tropical rain forest river in Costa Rica. Revista de Biología Tropical. 4: 791-809.

Cala P.1990. Diversidad, adaptaciones ecológicas y distribución geográfica de las familias de peces de agua dulce de Colombia. Revista de la Academia Colombiana de Ciencias Físicas y Exactas. XVII (67): 726-740.

Cardona M., Román-Valencia C., Jiménez J. y Hurtado H. 1998. Composición y diversidad de la quebrada San Pablo en Alto Cauca, Colombia. Boletín Ecotrópica. (32). 11-24.

CEIFI. 2003. Grupo de Investigaciones de Ingeniería Civil Universidad del Quindío. Modelación de Corrientes Hídricas Superficiales en el Departamento del Quindío. Fase II. Informe final de la Modelación de la Quebrada Cristales.

Cuartas D. 2005. Ecología trófica y reproductiva de "Poecilia caucana" (especie nativa) y "Xiphophorus helleri" (especie exótica) (Pices: Poeciliidae), en la cuenca alta del rio La Vieja, Colombia. Trabajo de grado, universidad del Quindío, Colombia. $172 \mathrm{p}$.

García-Alzate C y Román-Valencia C. 2008. Biología alimentaria y reproductiva de Hyphessobrycon poecilioides (Pisces:Characidae) en Alto Cauca, Colombia. Revista del museo Argentino de Ciencias Naturales n.s, 10(1): 17-27. 
García-Alzate C., Román-Valencia C., Vanegas-Rios A., y Arcila-Mesa D. 2007. Análisis fisicoquímico y biológico comparado en dos quebradas de alta montaña neotropical. Revista de Investigaciones de la Universidad del Quindio. 17:57-80.

Jiménez J., Román-Valencia C. y Cardona M. 1998. Distribución y constancia de las comunidades de peces del río San Pablo, Cuenca del río la Paila, Alto Cauca, Colombia. Revista Actualidades Biologicas, 20(68):21-27.

Lehmann P. 1999. Composición y estructura de las comunidades de peces de dos tributarios en la parte alta del río Cauca, Colombia. Cespedesia, 23(73-74):9-45.

Lowe-McConnell R. 1987. Fish Communities in tropical freshwaters. Cambridge University Press, Cambridge, 382p.

Machado-Allinson A. 1987. Los peces de los Llanos de Venezuela, un ensayo sobre su historia natural. Universidad Central de Venezuela Caracas. 105p.

Maldonado-Ocampo J., Ortega-Lara A., Usma J. S., Galvis G., Villa-Navarro F., Vásquez L., Prada-Pedrero S. y Ardila C. 2005. Peces de los Andes de Colombia. Instituto Alexander von Humboldt. Bogota, Colombia. 346 p.

Margalef R. 1982. Ecologìa. Editorial Omega.S.A. Barcelona. 951 p.

Nagles P., Vargas P. 2003. Diagnostico de la quebrada Cristales con macroinvertebrados acuáticos como indicadores biológicos en Armenia-La Tebaida, Quindío. Trabajo de grado, universidad del Quindío, Colombia. 106 p.

Nebiolo E. 1987. Composición y estructura de la ictiofauna del río Chama, Mérida Venezuela. Boletin de la Sociedad Venezolana de Ciencias Naturales, Tomo XLI (144): 167-184.

Noss R. 1990. Indicator for monitoring biodiversity: a hierarchical approach. Conservation biology 4:355-364. Ortega-Lara A., Usma J., Bonilla P., Santos N. 2006. Peces de la cuenca alta del río Cauca, Colombia. Biota Colombiana, 7(1):39-54.

Román-Valencia C. 1993. Composición y estructura de las comunidades de peces de la cuenca del río La Vieja, Alto Cauca, Colombia. Biología y Educacion, 3(5): 8-19.

Román-Valencia C. 1988. Clave taxonómica para la determinación de peces nativos del departamento del Quindío, subsistema alto río Cauca, Colombia. Actualidades Biológicas. 17(64): 107-114.

Román-Valencia C. 1995. Lista anotada de los peces en la cuenca del río La Vieja, Alto Cauca, Colombia. Boletín Ecotrópica: Ecosistema Tropicales (29): 11-22.

Román-Valencia C. 2003. Sistemática de las especies colombianas de Bryconamericus (Characiformes: Characidae) Dahlia, Revista de la Asociación Colombiana de Ictiólogos. 6: 17-58.

Román-Valencia C., Lehmann P. y Rubio E. 1999. Distribución y constancia de los peces del río San Miguel y el zanjón Bagazal en el Alto río Cauca, Colombia. Revista Actualidades Biologicas. 21(71): 163-172.

Román-Valencia C. y Perdomo A. 2004. Ecología trófica y reproductiva de Argopleura magdalenensis (Pisces: Characidae) en la cuenca alta de los ríos Cauca y Magdalena, Colombia. Revista del Museo Argentino de Ciencias Naturales, 6(1): 175-182.

Román-Valencia C., Botero A. y Ruiz R. 2003. Trophic and reproductive ecology of Roeboides dayi (Teleostei: Characidae) from upper Rio Cauca, Colombia. Bolletim Museum Regional Science Natural de Torino, 20(2): 487-496.

Ruiz-C R. y Román-Valencia C. 2006a. Osteología de Astyanax aurocaudatus, Eigennman, 1913 (Pisces: Characidae), con notas sobre la validez de Carlastyanax, Gèry, 1972. Animal Biodiversity and Conservation. 29(1): 49-51.

Ruiz-CR. y Román-Valencia C. 2006b. Aspectos taxonómicos de Cetopshorhamdia boquillae y C. nasus (Pisces: Heptapteridae), con anotaciones sobre su ecología en al cuenca alta de los ríos Magdalena y Cauca, Colombia. Animal biodiversity and Conservation, 29(2): 123-131.

Samudio H. 2004. Aspectos taxonómicos y ecológicos de Lasiancistrus caucanus (Pisces: Loricariidae) en la cuenca del río La Vieja, Alto Cauca, Colombia. Trabajo de grado, Universidad del Quindío, Armenia, Colombia.

Sánchez-Botero J., Lima de Farias M., Piedade M. y Sequeira D. 2003. íctiofauna asociada ás macrófitas aquáticas Eichhornia azurea (SW:) Kunth. E Eichhornia crassipes (Mart.) Solms. No loga Camaleão, Amazõnia Central, Brasil. Acta Scientirum. Biological Sciences. Maringa, 25(2): 369-375.

Uieda V. 1984. Ocorrência e distribuição dos piexes em nacho de agua doce. Revista Brasileira de Biologia 44(2): 203-213.

Vargas I. 1997. Inventario preliminar de los macroinvertebrados bentónicos en el río Quindío y la quebrada Cristales. C.R.Q., Armenia, $34 \mathrm{p}$.

Wetzel R. y Likens G. 2000. Limnological analyses, 3 ed. Nueva York: Springer-Verlag. 429 p.

Wooton J. 1992. Fish ecology. Chapman and Hall. New York, pp 59-111. 